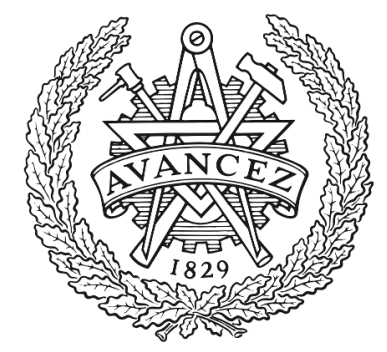

CHALMERS

UNIVERSITY OF TECHNOLOGY

\title{
Morphological Computing in Cognitive Systems, Connecting Data to Intelligent Agency
}

Downloaded from: https://research.chalmers.se, 2023-04-26 13:33 UTC

Citation for the original published paper (version of record):

Dodig Crnkovic, G. (2020). Morphological Computing in Cognitive Systems, Connecting Data to Intelligent Agency. Proceedings (MDPI), 47(1). http://dx.doi.org/10.3390/proceedings2020047041

N.B. When citing this work, cite the original published paper. 


\title{
Morphological Computing in Cognitive Systems, Connecting Data to Intelligent Agency ${ }^{\dagger}$
}

\author{
Gordana Dodig-Crnkovic \\ Department of Computer Science and Engineering, Chalmers University of Technology, 41296 Gothenburg, \\ Sweden; gordana.dodig-crnkovic@chalmers.se \\ † Fourth International Conference on Philosophy of Information, Berkeley, CA, USA and 2-6 June 2019. \\ Published: 15 May 2020
}

\begin{abstract}
This paper addresses some of the major controversies underlying the theme of the IS4SI 2019 Berkeley summit: "Where is the I in AI and the meaning of Information?". It analyzes the relationship between cognition and intelligence in the light of the difference between old, abstract and the new embodied, embedded, enactive computationalism. It is questioning presuppositions of old computationalism which described the abstract ability of humans to construct knowledge as a symbol system, comparing it to the modern view of cognition found in various degrees in all living beings, with morphological/physical computational processes emerging at a variety of levels of organization. Cognitive computing based on natural/ physical/ morphological computation is used to explain the goal-directed behavior of an agent acting on its own behalf (the "I" as self-referential awareness) applicable to both living beings and machines with varying degrees of intelligence.
\end{abstract}

Keywords: intelligence; cognition; artificial intelligence; information processing; morphological computing; natural computing; unconventional computing; evolution of agency

\section{Life, Agency, Cognition, Intelligence}

Each living being, starting from the simplest living cells, is an agent with the ability to generate behavior favorable for its own survival and thriving based on the interactions with its environment, including other living agents [1]. Within the framework of info-computationalism, in which information, always embodied in physical substrate [2], represents the world for an agent, while physical/embodied/morphological computation stands for all dynamics processes over informational structures, [3-5] living being are necessary consequence of the properties of physical matter that historically self-organized starting with a biogenesis leading to first living creatures, that continued evolving driven by own internal agency. Morphology (shape/structure + material properties) of an agent (a living organism or a machine) enables and constrains its possible interactions with the environment as well as its development, including its growth and reconfiguration [6]. Evolution explained info-computationally with information and (morphological) computation at a variety of levels of organization is slightly different from the classical view of random mutations + selection as mutations are not governed only by chance but also by morphology and agency of a living organism. It is along the lines of the modern extended evolutionary synthesis [1] (pp. 103-113), [7,8], with its constructive development and reciprocal causation (as a consequence of interaction).

In the beginnings of cognitive science, cognition was considered as a connection between information and knowledge, without elaboration either on the processes that precede the acquisition of information or those which come after knowledge construction by abstract (substrateindependent) computation. This old view of (disembodied) cognition thus leaves bodily aspects out of the scope of cognitive science. It is directly connected with old computationalism - symbol manipulation view of cognition. Even though the new computationalism [4,9] also builds on information processing, it takes into account the embodiment through a morphological model of 
computation as a physical process. The meaning of information is not substrate independent in the new info-computational (agent-based) approach, but it is relative to the agent. The same physical input (e.g., light) have a different meaning for a Cyanobacterium (as an energy source which via photosynthesis generates chemical energy that drives its activity) or a human (as a basis of vision).

Inspired by the models of "minimal cognition" and levels of cognition [4] in living organisms, computational and robotic cognitive systems have been developed with varying degrees of cognition and intelligence. Studies of cognition and intelligence in other, simpler living beings, from the simplest ones like viruses that act as molecular machines to unicellular organisms like bacteria and up in the level of complexity show a clear connection between the physical embodiment and cognition or intelligence. Certain functions of contemporary cognitive computing and AI surpass humans (calculation, search, memory, in some cases processing speed and even sensor and actuator capabilities) but many others are far below the human level, such as common-sense reasoning. Intelligence is a capacity closely connected with cognition, where cognition is understood as a process of "being in the world" of an agent. For living organisms cognition = process of life (perception, internal process control by information, actuation/agency) as argued byStewart, [10].

Intelligence is a specific ability of problem-solving and learning adaptive behaviors of an agent within an environment/context. In that sense, it is a subset/tool of cognition. Artificial Intelligence (AI) traditionally has been trying to reproduce human intelligent behavior in the computing machinery based on logical reasoning. Both traditional "good old" cognitivism and AI have been envisaged as results of logical operations, either on the information obtained directly from the perception and stored in the memory in humans, or from databases and online searches in the machinery. However, as the quick reaching of the limits of GOFAI (good old-fashioned AI) project have shown, cognition and intelligence in humans are substantially dependent on their embodiment, [11], thus not only logical processes of reasoning, but also on their form (morphology) on different levels of organization, physics and chemistry (thus details of implementation of information processing mechanisms).

\section{New Embodiment-Based Understanding of Computational Cognition and Intelligence}

Already Braitenberg [12] and Brooks [13] have shown that goal-directed behavior can be generated in a machine without representation/symbol manipulation. New developments in Machine Learning and especially Deep learning have contributed to the shift of focus in AI from reasoning and planning (symbolic computation) to learning from the data. The data sensing, gathering and processing is the first step in the cognition of living organism as well. Data as atoms of information, are used to learn about the world without the symbolic layer of computation. Deep learning AI has been shown among others capable of learning behaviors such a playing chess or go far better than humans.

New developments of computational approaches to cognition and intelligence, especially in robotic implementations show that the body is an integral part of cognitive processes and computation is not only symbol manipulation, but also physical processes in the body of cognizing agent know as morphological computation [14]. Those new models of computation appear under a variety of names such as natural computing/computing nature, unconventional computing, morphological computing, physical computing etc. I will highlight differences between cognitive computing and artificial intelligence and their connections to cognition and intelligence in nature within the framework of computing nature.

Traditionally, in philosophy, psychology and cognitive science cognitive and intelligent agents were always conceived as (grown-up, neurotypical) humans. Today, with new insights into deep details and mechanisms of cognition, insight is crystallizing that human cognition and intelligence are based not only in activities of brain and nerve cells, but also emerges from the interaction of the body with the environment, and the body itself with all the cells communicating with the neurons and the brain. Equally important is a new understanding of cognitive (sensory-based) and intelligent (problem-solving) processes that regulate the state of the cell. In other words, both concepts of 
cognition and intelligence have increased in scope with a new conception of their underlying mechanisms - from the activity on the level of the human brain, to the process on the cell level.

\section{Natural, Physical, Analog and Morphological Computation: From Data to Intelligent Agency}

Information in the structure of the world [15] is transformed by cognizing agents into data, information and knowledge [16]. In the info-computational approach to cognitive agency, data and information are building blocks that control processes in living cells, [4,5]. Morphological computation is (physical) information processing on different levels of organization in physical systems. The essential property of morphological computation is that it is defined on a structure of nodes (physical agents) that exchange (communicate) information. Unicellular organisms such as bacteria communicate and build swarms with far more advanced capabilities then individual organisms, through computational social/distributed cognition realized by information exchange. In general, groups of smaller organisms (cells) in nature self-organize into multicellular assemblies with differentiated control mechanisms from the cell level to the more complex structures of tissue, organ, organism and groups of organisms, and this layered organization provides information processing benefits.

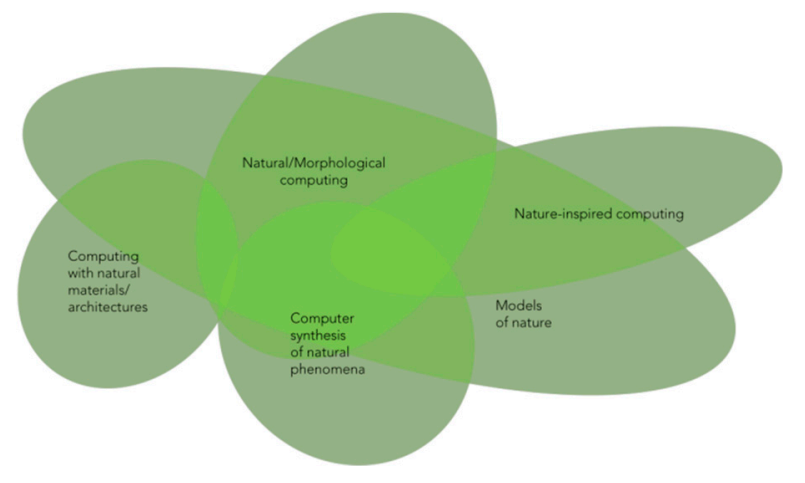

Figure 1. Morphological computing in its context.

In robotics, morphological computation is connecting the body, brain, and environment [11]. "Brain and body", which roboticists learn from, can belong to an octopus. Related research fields comprise among others: soft robotics, self-assembly systems, molecular computing, reservoir computing, spatio-temporal dynamics, information theoretical embodiment, amorphous computing, molecular computing and other kinds of morphological, natural, physical, unconventional computing [17-19]. A living agent is driven by its current morphology under given environmental and internal constraints to new behaviors (short term) and new morphologies (longer-term development \& evolution). In a mathematical formulation of info-computational model Ehresmann [20] shows how (neuro-)cognitive systems could be capable of creativity, in layered computational architecture.

\section{Conclusions}

Connecting data to intelligent agency is done by modeling cognitive systems (natural and artifactual) as information processors, equipped with physical information sensors that take in data (atomic information), process it through data/information self-organization that affects physical actuators, acting upon information through processes of morphological computation on different levels of organization of the cognizing agent. Underlying assumptions are as follows:

1. Nature is modeled as a network of networks of info-computational processes on several levels of organization. There is no information and thus no computation without physical implementation.

2. Dynamics of natural $/$ physical information $=$ physical $/$ natural $/$ morphological computation

3. Cognition and its subset intelligence $=$ natural $/ \mathrm{morphological}$ computation in cognitive agents 
The above assumptions lead to the following implications:

1. Cognition and intelligence are abilities of all living beings that evolve from abiogenesis via evolution of living agents and they come in degrees

2. Cognition and intelligence are both EEEE (Embodied, Embedded, Enactive, Extended)

3. For living organisms Cognition=Life. For artifacts, Cognition $=$ Artifactual surrogate of life

4. Intelligence $=$ Problem-solving ability, for both living organisms and artifacts. Its basic precondition is the ability to learn (biological or machine learning)

5. All cognizing systems (organisms and artifacts) are essentially dependent on information input from their surrounding and internal structures

6. Biological cognition and intelligence can only be understood in the context of their constant development and evolution driven by the interactions, internal and external

7. Evolution in the computing nature is the result of morphological computation on the succession of levels of organization (physical, chemical, biological, cognitive, social). That is reflectedon the genetic, epigenetic, behavioral, and symbolic levels, as extended evolutionary synthesis.

Funding: This research is funded by Swedish Research Council, VR grant.

Conflicts of Interest: The author declares no conflict of interest.

\section{References}

1. Torday, J.; Miller, W., Jr. Cellular-Molecular Mechanisms in Epigenetic Evolutionary Biology; Springer Nature Switzerland AG: Basel, Switzerland, 2020

2. Landauer, R. The physical nature of information. Phys. Lett. A 1996, 217, 188-193.

3. Dodig-Crnkovic, G. Nature as a Network of Morphological Info-computational Processes for Cognitive Agents. Eur. Phys. J. 2017, 226, 181-195, doi:10.1140/epjst/e2016-60362-9.

4. Dodig-Crnkovic, G. Modeling Life as Cognitive Info-Computation. In Proceedings of the 10th Computability in Europe, Budapest, Hungary, 23-27 June 2014; Csuhaj-Varjú, E., Meer, K., Eds.; Springer Nature Switzerland: Basel, Switzerland, 2014.

5. Dodig-Crnkovic, G. Information, Computation, Cognition. Agency-Based Hierarchies of Levels. In Fundamental Issues of Artificial Intelligence; Muiller, V.C., Ed.; Synthese Library 377; Springer Int. Pub.: Basel, Switzerland, 2016. doi:10.1007/978-3-319-26485-1_10.

6. Dodig-Crnkovic, G. The Info-computational Nature of Morphological Computing. In Theory and Philosophy of Artificial Intelligence; Müller, V.C., Ed.; Springer: Berlin, Germany, 2013; pp. 59-68.

7. Jablonka, E.; Lamb, M.J. Evolution in Four Dimensions, Revised Edition. Genetic, Epigenetic, Behavioral, and Symbolic Variation in the History of Life; The MIT Press: Cambridge, MA, USA, 2019.

8. $\quad$ Laland, K.N.; Uller, T.; Feldman, M.W.; Sterelny, K.; Müller, G.B.; Moczek, A.; Jablonka, E.; Odling-Smee, J. The extended evolutionary synthesis: its structure, assumptions and predictions. Proc. R. Soc. B 2015, 282, doi:10.1098/rspb.2015.1019.

9. Scheutz, M. Computationalism: The next generation. In Computationalism: New Directions; MIT Press: Cambridge, MA, USA, 2002; pp. 517-524.

10. Stewart, J. Cognition = Life: Implications for higher-level cognition. Behav. Process. 1996, 35, 311.

11. Pfeifer, R.; Bongard, J. How the Body Shapes the Way We Think: A New View of Intelligence; MIT Press: London, UK, 2006.

12. Braitenberg, V. Vehicles: Experiments in Synthetic Psychology; MIT Press: London, UK, 1986.

13. Brooks, R.A. Intelligence without representation. Artif. Intell. 1991, 47, 139-159.

14. Paul, C. Morphology and Computation. In Proceedings of the International Conference on the Simulation of Adaptive Behavior, Los Angeles, CA, USA, 13-17 July 2004; pp. 33-38.

15. Burgin, M. Data, Information and Knowledge. Information 2004, 7, 47-57.

16. Burgin, M. Information in the structure of the world. Inf. Theor. Appl. 2011, 18, 16-32.

17. Hauser, H.; Füchslin, R.M.; Nakajima, K. Morphological Computation-The Physical Body as Computational Resource. In Opinions and Outlooks on Morphological Computation; University of Bristol: Bristol, UK, 2014; Chapter 20; ISBN 978-3-033-04515-6. 
18. Adamatzky, A. Advances in Unconventional Computing; Springer: Berlin, Germany, 2016; Volume 1.

19. Mikkilineni, R. Going beyond computation and its limits: Injecting cognition into computing. Appl. Math. 2012, 3, 1826-1835.

20. Ehresmann, A.C. MENS, an Info-Computational Model for (Neuro-)cognitive Systems Capable of Creativity. Entropy 2012, 14, 1703-1716. article distributed under the terms and conditions of the Creative Commons Attribution (CC BY) license (http://creativecommons.org/licenses/by/4.0/). 